\title{
Auxiliary Decision Support Model of Sports Training Based on Association Rules
}

\author{
Changnian Zhang, ${ }^{1}$ MeiJie Li $\mathbb{D D}^{2,3}$ Hui Wang ${ }^{\mathbb{D}},{ }^{4}$ and Ning Wang ${ }^{4}$ \\ ${ }^{1}$ School of Dance and Martial Arts, Capital University of Physical Education and Sports, Beijing 100191, China \\ ${ }^{2}$ Humour Division, Huanghe University of Science and Technology, Zhengzhou 450063, China \\ ${ }^{3}$ Sports Institute, Korea Gangneung-Wonju National University, Gangneung 25457, Republic of Korea \\ ${ }^{4}$ Faculty of Physical Culture, Gdansk University of Physical Education and Sport, Gdansk 80-336, Poland \\ Correspondence should be addressed to Meijie Li; 201508113@hhstu.edu.cn
}

Received 28 May 2021; Accepted 13 July 2021; Published 27 July 2021

Academic Editor: Fazlullah Khan

Copyright @ 2021 Changnian Zhang et al. This is an open access article distributed under the Creative Commons Attribution License, which permits unrestricted use, distribution, and reproduction in any medium, provided the original work is properly cited.

\begin{abstract}
In sports or fitness training, nonstandard movements will affect the training effect and even lead to sports injuries. However, the standard movements of various sports activities need professional guidance, so it is difficult to find out whether the movements are standard or not. In recent years, body pose estimation has become a hot topic in computer vision research. A deep learning model can effectively identify the human nodes and movement trajectory in pictures or videos and evaluate the movements of the target human body. However, the movement process is generally covered by others or the situation of nearby personnel, which leads to the deviation of the movement recognition of the human body and affects the evaluation of the movement. Thus, it is unable to effectively correct the wrong movement, but will mislead the training personnel. Therefore, this paper proposes a novel decision support model for sports training based on association rules. We use posterior probability settings to reveal the weights of the discriminative ability of attribute items and set the classification performance to reflect the weights of three measures to evaluate credit contribution. Thus, the learning threshold setting reflects the weight of the decision-making ability of sports training. Furthermore, compared with traditional association rules, attribute items, frequent item sets, and classification rules that can improve the decision-making performance of sports training are discovered, which complement the deficiencies of different measures. Finally, using the weighted voting strategy to fuse the decision-making information of the classification rules, we can effectively assist in sports training so that the coach can work out corresponding countermeasures and realize scientific management.
\end{abstract}

\section{Introduction}

When people participate in sports training [1-3], sports instructors [4-6] play a vital role. The instructor is responsible for the movement guidance, health care [7-9] guidance, and health assessment $[10,11]$ of sports activities and various exercises. Due to the rigorous involvement of sports instructors, athletes can master scientific exercise methods and correct techniques, which eventually and effectively improve the level of exercise and achieve sports training. However, the number of sports instructors and the number of exercises in our country live far from each other, causing many exercisers and athletes in our country to lack professional guidance. Even in physical education classes [12-14], it is difficult for teachers to pay attention to every student's move during exercise. Suppose the movement is wrong during sports training. In that case, it will fail to achieve the exercise effect and even be harmful to the body. Therefore, there is an urgent need for intelligent sports training supervision and guidance programs.

With the revolutionary progress in the field of artificial intelligence [15-19] research, it has become possible to use intelligent machines to replace humans to perform simple and repetitive tasks, serve human life, and liberate productivity. Human-computer interaction is a hot topic of artificial intelligence technology research. However, it is not a simple matter 
to allow machines to learn and understand our human instructions and communicate with humans. The process of human-computer interaction requires machines to recognize and complete human instructions and work accordingly accurately. Human body pose estimation technology is currently an effective way to realize human-computer interaction [9]. However, it is common for other people to visually obstruct the subject during the exercise process, which in turn leads to deviations in recognition of human actions, which then affect the evaluation of the actions and ultimately mislead trainers to correct the wrong actions effectively.

At present, the standard of movement is judged by naked eye observation. The shortcomings of this judgment method are low efficiency, too subjective, lack of objectivity, and lack of unified quantitative indicators $[10,11]$. There is little research on computer vision technology application in sports training [16-19]. It only uses artificial intelligence technology to recognize athletes' movements to obtain athletes' body posture information. However, there is no standard, and no work has been found on the issue when other foreign objects block a part of the athlete's body or the movement is highly symmetrical. The current network structure will not be able to accurately recognize the athlete's body posture and thus cannot be evaluated and analyzed. Such recognition results will have many limitations.

The decision support system is an interactive computer system that can provide decision makers with valuable information and an environment for creative thinking and learning and help decision makers solve semistructured and unstructured decision-making problems. Its merits are mainly reflected in its support for the whole process of decision-making, especially its ability to support each stage of the decision-making process. This undoubtedly provides powerful means to control the sports training process.

This paper constructs a sports training decision support model, combining association rules and adaptive weighted decision fusion based on the above observation. Association rule mining based on a priori is a process to obtain strong association rules by mining frequent item sets in the data set according to a given measure (support, confidence, or weighted chi-square). However, different attribute items, measures, and rules have different discriminating abilities to sports training decisions. Therefore, this paper uses a posteriori probability to set the weight to reveal the discriminant ability of attribute items, embodies the weight to evaluate the credit contribution degree of the three measures through the classification performance setting, and reflects the weight to evaluate the credit contribution degree of the rules through the learning threshold setting. Furthermore, compared with traditional association rules, attribute items, frequent item sets, and classification rules can improve the performance of sports training decisionmaking better, complementing the shortcomings of different measures. Finally, the weighted voting strategy is used to fuse the decision information of classification rules.

The following are the main contributions of this paper:

(1) In this paper, the posterior probability is used to set the weight of the discriminative ability of the attribute item. The weight of the three measures to evaluate the credit contribution degree is reflected by the classification performance setting. The threshold of learning sets the weight of the credit evaluation ability of the rule. Furthermore, compared with traditional association rules, attribute items, frequent item sets, and classification rules that can improve the decision-making performance of sports training are discovered, which complements the deficiencies of different measures.

(2) This paper uses association rules to reduce the dimensionality of high-dimensional data, realizes decision information fusion through five similarity measures, and builds a sports training auxiliary decision support model that combines multiview similarity measures and association rules.

\section{Related Work}

Human research in the field of human motion analysis technology has gone through a long process. Its history is much earlier than modern computer vision [20-22] technology. It can even be traced back to the fifteenth century. In order to better depict specific movements of the human body and create lifelike works, Leonardo da Vinci, a world-famous painter, has done some work on the force of the limbs and the state of muscle tissue when the human body performs these movements. In the nineteenth century, the French scientist Mahler invented the "Mahler Photographic Gun." This invention enabled continuous photography to move from dream to reality. It can show moving objects on the same film. During this period, British videographer Muybridge developed an animal experiment mirror, which can achieve rapid and continuous projection of moving objects. They have all made many contributions towards recording human movement.

Some research work on motion perception opened the prelude to the use of image sequences to study human motion. In his experiment, he put LED lights on the human body joints (such as wrists, ankles, and knees). He then made the human body perform actions in a dark environment. In this case, only the relevant nodes are visible. Recording the position and combination of light spots during exercise is equivalent to recording the movement of the human body. Its experimental results show that these light spot combinations can distinguish human movement. Later, with developing disciplines such as computer vision, artificial intelligence, pattern recognition, and image processing, these disciplines have also joined the research team on human motion analysis.

The study of human motion is a typical research problem of nonrigid body motion. Although there is a relatively complete and comprehensive research theory for rigid body motion research, the research on nonrigid body motion of a typical nonrigid structure like human motion is still in a relatively preliminary stage. Compared with the depth of people's research in the field of rigid motion, people still have a long way to go for the motion analysis research technology of nonrigid structures. In human motion analysis research, model-based human motion analysis methods 
need to rely on prior knowledge about the human body. When using this method for research, a 2D or 3D model of the human body must be established first. The feature vector of the model-based human motion analysis method is mainly obtained from the model, and the establishment of the human body model is a relatively complicated process, so it is less used in practical applications. The model-free method does not require the establishment of a human body model. This method directly extracts features from the image sequence to describe human actions. Commonly used features include contours, area perimeter ratios, and trajectory regions of interest, which are generally specific. The corresponding feature descriptor is extracted from the recognition behavior, and then, the corresponding feature space is established as the expression of the behavior to be recognized in the computer. At present, the model-free method is widely used in the research of human behavior recognition. Many scholars at home and abroad have been conducting much scientific research on extracting highly robust feature vectors and efficient recognition algorithms [23] easily. In summary, this article adopts an auxiliary decision-making model for sports training based on association rules.

\section{Methodology}

This paper uses the Hotelling $T^{2}$ statistic to measure the similarity of human motion from the perspective of parameter hypothesis testing, selects the Jensen-Shannon difference to measure the similarities and differences of the sports training effect from the perspective of the information contained in the distribution, and selects the cosine distance to measure the motion from the perspective of the vector direction the difference in training effect.

3.1. Similarity Measure. The Hotelling $T^{2}$ statistic is a generalization of the multivariate $T$-ratio distribution. In fact, the square of $T$-ratio for univariate data is a special case of Hotelling $T_{z}$ distance. It has many acquaintances with Mahalanobis distance. The calculation equation is

$$
D=\sqrt{\frac{T^{2}\left(n_{1}+n_{2}\right)}{n_{1} n_{2}}}=\sqrt{(\bar{X}-\bar{Y})^{\prime} S^{-1}(\bar{X}-\bar{Y}) .}
$$

Hotelling $T^{2}$ is a unitless value that uses a single standard deviation to describe the distance between two samples. Among them $n_{1}$ and $n_{2}$ are sample sizes and $\bar{X}$ and $\bar{Y}$ are sample averages. The Hotelling $T^{2}$ statistic is a unitless metric that is often used to measure data changes, and it is also suitable for high-dimensional data.

The Jensen-Shannon difference is also called (JensenShannon divergence), which measures the similarity between two probability distributions. It solves the problem of asymmetry of KL divergence. The J-S divergence is symmetric, and its value is between 0 and 1 . It is defined as follows:

$$
\mathrm{JS}(p \| q)=\frac{1}{2} \mathrm{KL}\left(p \| \frac{p+q}{2}\right)+\frac{1}{2} \mathrm{KL}\left(q \| \frac{p+q}{2}\right),
$$

where $p$ and $q$ are two distributions.
The cosine of the angle in the geometry of the cosine distance can be used to measure the difference between two vector directions. This concept is used in machine learning to measure the difference between sample vectors. The cosine distance in $n$-dimensional space is as follows:

$$
D=\frac{x \cdot y}{\|x\|\|y\|},
$$

where $x$ and $y$ are $n$-dimensional vectors.

3.2. Weighted Fusion. This paper proposes a decision fusion algorithm for the fusion of multiple similarity measures. The important idea is to use multiclassifier fusion technology to perform weighted voting fusion on decision information of three different similarity measures: Hotelling $T^{2}$ statistic, Jensen-Shannon difference, and cosine distance. Given the matrix $L$,

$$
L=\left[\begin{array}{cccc}
l_{11} & l_{12} & \cdots & l_{1 n} \\
l_{21} & l_{22} & \cdots & l_{2 n} \\
\vdots & \vdots & \ddots & \vdots \\
l_{m 1} & l_{m 2} & \cdots & l_{m n}
\end{array}\right]_{m \times n} .
$$

It consists of class labels determined by a single classifier, where $l_{i j}$ represents the classification label of sample $j$ using classifier $i, N$ is the category number, $m$ is the total number of samples, and $n$ is the number of classifiers. The weight $w_{i}$ of the classifier $i$ represents its classification performance for $m$ samples:

$$
\begin{aligned}
w_{i} & =\frac{\sum_{j=1}^{m} \delta\left(l_{i j}, y_{j}\right)}{m}, \quad i=1,2, \ldots, n, \\
\delta(k, g) & = \begin{cases}1, & \text { if } k=g, \\
0, & \text { else. }\end{cases}
\end{aligned}
$$

In order to make the final decision on the label prediction, the voting decision matrix is defined as $V$ :

$$
V=\left[\begin{array}{cccc}
v\left(l_{11}\right) & v\left(l_{12}\right) & \cdots & v\left(l_{1 \mathrm{n}}\right) \\
v\left(l_{21}\right) & v\left(l_{22}\right) & \cdots & v\left(l_{2 \mathrm{n}}\right) \\
\vdots & \vdots & \ddots & \vdots \\
v\left(l_{\mathrm{m}_{1}}\right) & v\left(l_{\mathrm{m}_{2}}\right) & \cdots & v\left(l_{\mathrm{mn}}\right)
\end{array}\right]_{m \times n} .
$$

Therefore, the final decision equation is the dot product of the weighted vector $W=\left[w_{1}, w_{2}, \ldots, w_{n}\right]$ and the voting decision matrix $V$ is as follows:

$$
D_{j}=[\underset{\text { column }}{\arg \max }[W \times V(j)],
$$

where $V(j)$ is the $j$ th column of the voting matrix $V$.

3.3. Association Rules. Association rules are a combination of association rule mining and classification technology and play an important role in the decision-making process of many applications and fields [24]. The purpose of association 
rule technology is to discover the correlation or association between items. In contrast, classification technology [25] is responsible for predicting class labels. For example, suppose the support or confidence of the generation rule is greater than or equal to the given minimum support or confidence. In that case, some items with strong correlation can be mined from the data, frequent item sets.

Let $T=\left\{t_{1}, t_{2}, \ldots, t_{n}\right\}$ and $X$ be $n$ different items $\left\{x_{1}, x_{2}, \ldots, x_{n}, c_{1}, c_{2}\right\}$ in the data set. $A \longrightarrow c$ is the classification association rule, where $A \subseteq X$ and $c$ means it belongs to the $\left\{c_{1}, c_{2}\right\}$ category. For example, the rule $A \longrightarrow c_{1}$ can be interpreted as if the attribute $A$ appears in the rule; then, we say that the rule belongs to the first category.

Shannon's diary is also called information diary, which reflects the relationship between the amount of information and the uncertainty of information. For example, given $x$, its Shannon's direct is

$$
H(x)=-\sum_{j=1}^{N} p_{j} \log _{2} p_{j},
$$

where $p_{j}$ represents the posterior probability of item $x, N$ represents the number of possible categories, and $H(x)$ calculates the amount of information of item $x$. The larger the entropy is, the smaller the amount of information of the item is and the weaker the item's ability to classify instances with item value $x$. Use $H(x)$ to calculate the assigned weight $W(x)$ of item $x$ :

$$
W(x)=\frac{\exp (-H(x))}{\sum_{x} \exp (-H(x))} .
$$

\section{Experiments and Results}

4.1. Experimental Setup. The hardware equipment tested in this article is a LENOVO 20DEAOIJCD, the processor is AMD A8-7100 Radeon R5, 8 Compute Cores 4C+4G, $1800 \mathrm{MHz}$, the memory is $8.0 \mathrm{OBB}$, and the hard disk storage is $1 \mathrm{~TB}$; a GPU is equipped with NVIDIA Tesla P40 Server, GPU architecture is NVIDIA Pascal, GPU memory is $24 \mathrm{~GB}$, single-precision floating-point computing capacity reaches 12 trillion floating-point operations per second, and maximum power consumption is $250 \mathrm{~W}$. The operating system is CentOS 7.5 (1804) and the development tool is matlab 2019, which is shown in Table 1.

4.2. Dataset. This paper uses GolfDB as the experimental data set, which is a high-quality golf swing video data set created specifically for golf swing motions and used for golf swing motion recognition. GolfDB contains 1,400 golf swing video samples, with a total of more than $390 \mathrm{~K}$ frames of video data, which are collected manually from YouTube video sites with 580 regular speed and slow-motion golf swing motion videos. It is the most important for golf swing motion recognition. To ensure that the club is visible and reduces motion blur, the sampling only considers $30 \mathrm{fps}$, $720 \mathrm{p}$ resolution video. The video mainly intercepts the swing movements of 248 professional golfers in the game video from PGA, LPGA, and Champions Tours obtained from different angles of different scenes of the golf course, such as driving range, tee, bunkers, and fairways. The dataset is freely available online at https://github.com/wmcnally/ GolfDB.

4.3. Data Augmentation. In view of the complex light source problems that may occur in the actual detection scene of the golf course and the target object scale problem caused by the different camera shooting angles and distances, this paper proposes a data enhancement scheme to expand the dataset so that the dataset can simulate various complex scenes and improve the generalization ability of the model in complex scenarios. For the open-air golf course, the strong sunlight source, as shown in Figure 1, adopts the method of increasing the image exposure value and the image contrast to simulate the overexposure environment of the camera.

4.4. Evaluation Methods. This paper uses precision, recall, and average precision to evaluate the performance of the model.

Precision is the degree to which measurements exhibit the same result when conditions are unchanged. It is calculated as the number of true positive divided by the number of true positive and false positive predictions and is calculated as

$$
P=\frac{\mathrm{TP}}{\mathrm{TP}+\mathrm{FP}}
$$

A recall is the fraction of all the positive predictions to overall positive examples in the dataset. It is a measure of sensitivity in binary classification. It is a probability of positive predictions retrieved by a classifier. A recall is calculated as follows:

$$
\text { Recall }=\frac{\mathrm{TP}}{\mathrm{TP}+\mathrm{FN}}
$$

Average precision is a measure that utilizes recall and precision and is the area under the precision and recall curve. Average precision (AP) is the average of (un-interpolated) precision values at all ranks where relevant documents are found. The calculation equation is as follows:

$$
\mathrm{AP}=\int_{0}^{1} P(\text { Recall }) d(\text { Recall }) \text {. }
$$

4.5. Experimental Results. The random sampling method of 1000 sample data is to select $10 \%$ of the sample population randomly for 10 times as training samples. This allows all samples in the population to have an equal chance of selection. The algorithm in this paper is compared with the five similarity measure methods, and the experimental results are shown in Figure 2.

From Figure 2, we can see that the recognition rates of the four similarity measures, Chebyshev distance, Hamming distance, cosine distance, and Jensen-Shannon difference, have increased slightly after processing the experimental 
TABLE 1: Server platform configuration.

\begin{tabular}{lc}
\hline Class & Parameter \\
\hline CPU & AMD A8-7100 radeon R5 \\
Memory & 8.00 GB \\
Hard disk & 1 TB \\
GPU & NVIDIA Tesla P40 \\
GPU memory & 24 GB \\
OS & CentOS 7.5 \\
Tool & Matlab 2019 \\
\hline
\end{tabular}

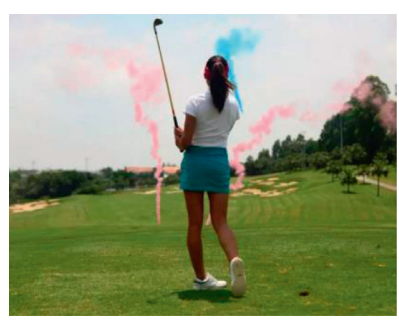

(a)

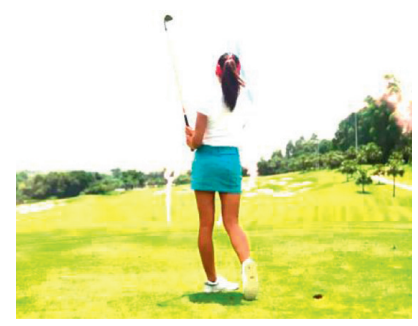

(b)

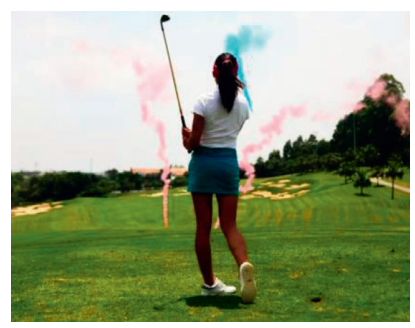

(c)

FIgURE 1: Data augmentation results. (a) Original image; (b) increase exposure; (c) increase contrast.

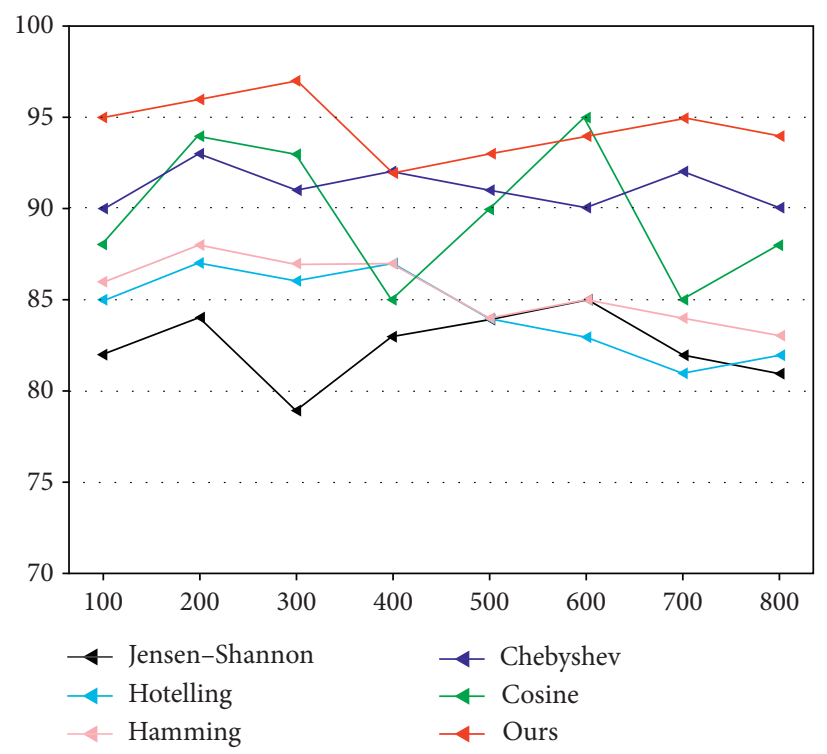

FIGURE 2: Comparative experimental results of different methods.

data, especially by the five similarities measurement methods. The recognition rate of the fusion algorithm in this paper has always been at a high level. When the training sample is less than 500 , the recognition rates of the three similarity measures of CFMSM-AR, Chebyshev distance, and Hamming distance have small fluctuations. In contrast, the other three lateral degree methods have large fluctuations. It can be seen and is evident from the result that our algorithm has better robustness.

Considering that the classification performance of the three measures, Support (SUP), Confidence (CONF), and weighted chi-square (WCH), is sometimes inconsistent, we take the weighted sum of measures as an integrated measure. In this experiment, we randomly selected a subset composed of 1000 data points from the original dataset. Then, we set the training sample ratio to $25 \%, 50 \%$, and $75 \%$ in three cases by randomly segmenting the training and test samples. Figures 3-5 show the classification accuracy of the four measures (SUP, CONF, WCH, and integration measure) obtained in these cases. In addition, the experimental results show that the proposed integration measure is always superior to the other three measures (SUP, CONF, and WCH). 


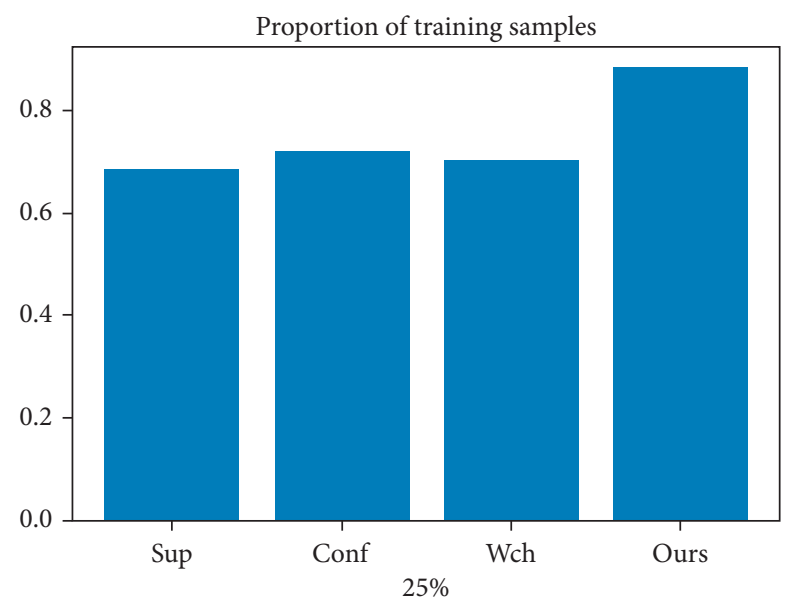

FIgURE 3: Proportion of training samples (25\%).

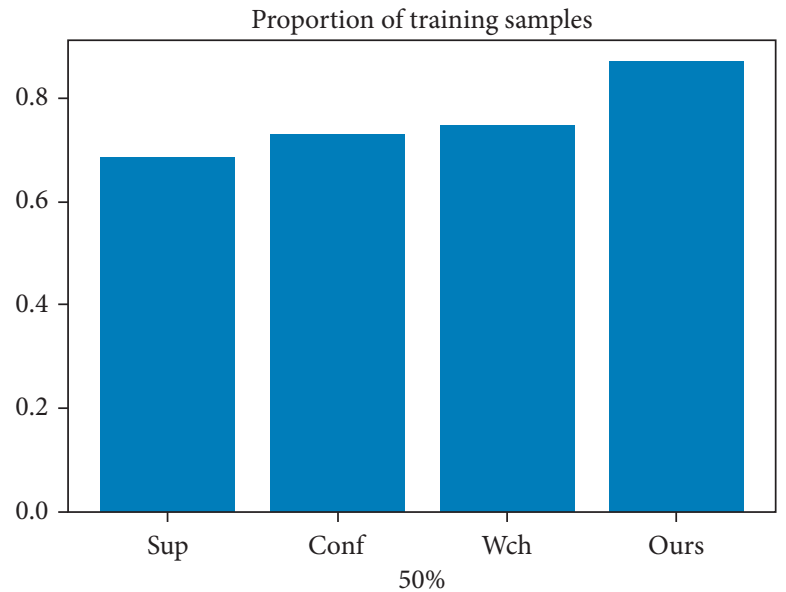

FIgURE 4: Proportion of training samples (50\%).

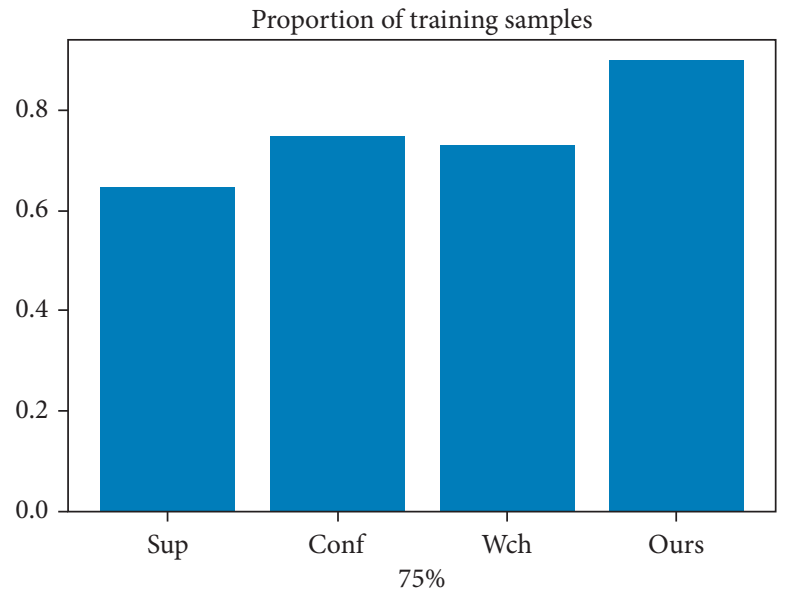

FIGURE 5: Proportion of training samples (75\%). 


\section{Conclusion}

This paper proposes a decision support model for sports training based on association rules. We use a posteriori probability setting to reveal the weights of the attribute items' discriminant ability and set the classification performance to reflect the weights of the three measures for evaluating credit contributions. The setting of the learning threshold reflects the importance of decision-making ability in sports training. In addition, compared with the traditional association rules, attribute items, frequent item sets, and classification rules are found to improve decision-making in sports training, which makes up for the shortcomings of different measures. Finally, the decision information of classification rules can be integrated with the weighted voting strategy, which can effectively assist the sports training, make the coaches work out the corresponding countermeasures, and realize the scientific management.

We understand that the current study's true advantage may be achieved by providing a web predictor that is available to the research community, coaches, and management for its utilization and further enhancement. Therefore, efforts will be made in the future to make this study available online.

\section{Data Availability}

The data used to support the findings of this study are included within the article.

\section{Conflicts of Interest}

The authors declare that they have no conflicts of interest.

\section{References}

[1] X. Wang, D. Huang, and X. Zhao, "Design of the sports training decision support system based on improved association rule, the apriori algorithm," Intelligent Automation \& Soft Computing, vol. 26, no. 4, pp. 755-763, 2020.

[2] K. Kasper, "Sports training principles," Current Sports Medicine Reports, vol. 18, no. 4, pp. 95-96, 2019.

[3] L. G. Appelbaum and G. Erickson, "Sports vision training: a review of the state-of-the-art in digital training techniques," International Review of Sport and Exercise Psychology, vol. 11, no. 1, pp. 160-189, 2018.

[4] N. Malkoc and S. Dal, "The relationship between perceived manager support and work commitments of sports instructors," Educational Research and Reviews, vol. 15, no. 7, pp. 395-402, 2020.

[5] D. Atilgan and Y. Tükel, "Examination of personal innovativeness perceptions and work engagement behaviors of sports instructors," International Journal of Applied Exercise Physiology, vol. 9, no. 9, pp. 90-104, 2020.

[6] J. Y. Son, "A study on influence of senior sports instructors' transformational leadership on organizational performance, job engagement, and innovative behavior," The Journal of the Korea Contents Association, vol. 19, no. 10, pp. 535-547, 2019.

[7] V. S. Naresh, S. S. Pericherla, P. Sita Rama Murty, and S. Reddi, "Internet of things in healthcare: architecture, applications, challenges, and solutions," Computer Systems Science and Engineering, vol. 35, no. 6, pp. 411-421, 2020.
[8] Y. Sun, Y. Yuan, Q. Wang, L. Wang, E. Li, and L. Qiao, "Research on the signal reconstruction of the phased array structural health monitoring based using the basis pursuit algorithm," Computers, Materials \& Continua, vol. 58, no. 2, pp. 409-420, 2019.

[9] E. Adel, S. El-sappagh, M. Elmogy, S. Barakat, and K. Kwak, "A fuzzy ontological infrastructure for semantic interoperability in distributed electronic health record," Intelligent Automation \& Soft Computing, vol. 26, no. 2, pp. 237-251, 2020.

[10] K. Kaur and K. Kaur, "Failure prediction, lead time estimation and health degree assessment for hard disk drives using voting based decision trees," Computers, Materials \& Continua, vol. 60, no. 3, pp. 913-946, 2019.

[11] S. Parvathavarthini, N. K. Visalaksi, S. Shanthi, and J. Mohan, "An improved crow search based intuitionistic fuzzy clustering algorithm for healthcare applications," Intelligent Automation \& Soft Computing, vol. 26, no. 2, pp. 253-260, 2020.

[12] G. Griban, O. Kuznietsova, D. Dzenzeliuk et al., Dynamics of Psycho-Emotional State and Individual Psychological Characteristics of Students in the Process of Physical Education Classes, Revista Dilemas Contemporáneos: Educación, Política y Valores. Año: VII Número: Edición Especial Artículo no. :113, 2019, http:// www.dilemascontemporaneoseducacionpoliticayvalores.com/.

[13] V. Kashuba, M. Kolos, O. Rudnytskyi et al., "Modern approaches to improving body constitution of female students within physical education classes," Journal of Physical Education and Sport, vol. 17, no. 4, pp. 2472-2476, 2017.

[14] G. Fin, J. A. Moreno-Murcia, J. León, E. Baretta, and R. J. N. Júnior, "Interpersonal autonomy support style and its consequences in physical education classes," PLoS One, vol. 14, no. 5, Article ID e0216609, 2019.

[15] L. Zhang, X. Wang, X. Dong, L. Sun, W. Cai, and X. Ning, "Finger vein image enhancement based on guided triGaussian filters," ASP Transactions on Pattern Recognition and Intelligent Systems, vol. 1, no. 1, pp. 17-23, 2021.

[16] R. Liu, X. Ning, W. Cai, and G. Li, "Multiscale dense crossattention mechanism with covariance pooling for hyperspectral image scene classification," Mobile Information Systems, vol. 2021, Article ID 9962057, 15 pages, 2021.

[17] Y. Tong, L. Yu, S. Li, J. Liu, H. Qin, and W. Li, "Polynomial fitting algorithm based on neural network," ASP Transactions on Pattern Recognition and Intelligent Systems, vol. 1, no. 1, pp. 32-39, 2021.

[18] Y. Zhang, W. Li, L. Zhang, X. Ning, L. Sun, and Y. Lu, "AGCNN: adaptive gabor convolutional neural networks with receptive fields for vein biometric recognition," Concurrency and Computation: Practice and Experience, Article ID e5697, 2020, In press.

[19] X. Zhang, Y. Yang, Z. Li, X. Ning, Y. Qin, and W. Cai, “An improved encoder-decoder network based on strip pool method applied to segmentation of farmland vacancy field," Entropy, vol. 23, no. 4, p. 435, 2021.

[20] Z. Huang, Y. Zhang, Q. Li et al., "Joint analysis and weighted synthesis sparsity priors for simultaneous denoising and destriping optical remote sensing images," IEEE Transactions on Geoscience and Remote Sensing, vol. 58, no. 10, pp. 6958-6982, 2020.

[21] Q. Liu, L. Cheng, A. L. Jia, and C. Liu, "Deep reinforcement learning for communication flow control in wireless mesh networks," IEEE Network, vol. 35, no. 2, pp. 112-119, 2021.

[22] J. Zhang, J. Sun, J. Wang, and X.-G. Yue, "Visual object tracking based on residual network and cascaded correlation 
filters," Journal of Ambient Intelligence and Humanized Computing, vol. 12, no. 8, pp. 8427-8440, 2020.

[23] Z. Huang, P. Zhang, R. Liu, and D. Li, "Immature apple detection method based on improved Yolov3," ASP Transactions on Internet of Things, vol. 1, no. 1, pp. 9-13, 2021.

[24] W. Cai, Y. Song, and Z. Wei, "Multimodal data guided spatial feature fusion and grouping strategy for E-commerce commodity demand forecasting," Mobile Information Systems, vol. 2021, Article ID 5568208, 14 pages, 2021.

[25] Z. Chen, J. Huang, H. Ahn, and X. Ning, "Costly features classification using monte carlo tree search," 2021, https:// arxiv.org/abs/2102.07073. 